\title{
Commentary \\ Acute kidney injury in the intensive care unit: current trends in incidence and outcome
}

\section{Dinna N Cruz ${ }^{1,2}$ and Claudio Ronco ${ }^{1}$}

\author{
1'Department of Nephrology, Ospedale San Bortolo, Vicenza, Italy \\ ${ }^{2}$ Section of Nephrology, Department of Medicine, St Luke's Medical Center, Quezon City, Philippines
}

Corresponding author: Dinna N Cruz, dinnacruzmd@yahoo.com

Published: 24 July 2007

This article is online at http://ccforum.com/content/11/4/149

(C) 2007 BioMed Central Ltd

Critical Care 2007, 11:149 (doi:10.1186/cc5965)

See related research by Bagshaw et al., http://ccforum.com/content/11/3/R68

\begin{abstract}
Acute kidney injury (AKI) is a common clinical problem with significant clinical and economic consequences. A number of studies point to a rising incidence of AKI in the hospital and in the intensive care unit over the past several years, and an increase in the degree of co-morbidity associated with it. Recent evidence suggests that there has been some improvement in outcomes over time. Nevertheless, the mortality associated with AKI remains unacceptably high, and further work is needed. Recently developed consensus definitions will be useful in this regard.
\end{abstract}

Bagshaw and colleagues [1] report on the epidemiology and outcomes of acute kidney injury (AKI) in Australian intensive care units (ICUs) over a ten year period. It has been said that despite technological advances in nephrology, there has been little improvement in the outcomes of patients with AKI [2]. The literature has been confounded by the use of varying definitions of $\mathrm{AKI}$, reliance on coding for $\mathrm{AKI}$ in administrative databases, and lack of adjustment for severity of illness and co-morbidities. Nevertheless, it is undisputed that there has been a notable increase in AKI incidence [3,4], and this has important economic implications.

The work by Bagshaw and colleagues [1] confirms the rising AKI incidence, but focuses on the critical care setting. Using a large multicenter ICU adult database, they noted that AKI incidence increased almost 3\% annually from 1996 to 2005. Since the ANZICS definition of AKI remained constant, their results are less likely to be affected by changes in coding practices over time. This Australian study now corroborates this 'epidemic' of AKI, at least in the ICU. As it is, this is an alarming trend. Furthermore, as they identified only AKI present within the first 24 hours of ICU admission, this underestimates the magnitude of the problem. Interestingly, the increase in $\mathrm{AKI}$ incidence does not appear to be entirely due to the older and sicker patients now in our ICUs, who are more prone to develop AKI. Indeed, the Acute Physiology And Chronic Health Evaluation (APACHE) score and Simplified Acute Physiology score (SAPS) of AKI patients have remained unchanged over the ten-year period. Instead, the trend for increasing $\mathrm{AKI}$ incidence is also seen in the less severely ill groups of patients: those with no co-morbid illness and elective ICU admissions. This may be in part related to the fact that the present study refers only to $\mathrm{AKI}$ on admission, and is based on blood creatinine levels. This criterion will tend to underdetect $\mathrm{AKI}$ in older patients with smaller muscle mass. In addition, it is possible that this group of patients develops 'delayed' $\mathrm{AKI}$, that is, after the first 24 hours of ICU admission. It has been suggested that this rise in $\mathrm{AKI}$ incidence is due to more aggressive diagnostic and therapeutic interventions in more recent years [5].

Reassuringly, however, we are seeing an apparent decline of early AKI in certain subgroups, such as hematological malignancy, trauma and cardiovascular surgery [1]. Even more encouraging is that there has been an apparent decrease over time in the mortality of AKI patients, with an annual decrease of $3.4 \%$ per year. This change persisted after adjustment for several factors, such as age, co-morbidity and severity of illness.

Although the ANZICS study is unable to provide us with the answers, we can speculate as to the possible reasons for this change. As suggested by the authors, this may be due to overall improvement of ICU care, as well as better collaboration between intensivists, nephrologists and other subspecialties. It is interesting, however, that there was no change in mortality over time in the non-AKI group, raising doubt that this is the only factor. It may well be that

$\mathrm{AKI}=$ acute kidney injury; AKIN = Acute Kidney Injury Network; ICU = intensive care unit; RIFLE = Risk-Injury-Failure-Loss of renal function-Endstage renal disease. 
improvements in dialytic care, with the now widespread use of biocompatible membranes, improved machinery and increasing attention to dose in both continuous and intermittent renal replacement therapies, contributed to better outcomes. This is congruent with a US study in which crude mortality in AKI that required dialysis decreased over a 15 year period [3]. Another potential explanation is the availability of less nephrotoxic alternatives for various drugs and contrast agents. This may also be related to a reduction in the use of old therapy mainstays such as 'renal dose dopamine' and diuretics which, under scientific scrutiny, have not been found to be effective. Perhaps this may be due to increased awareness and recognition of AKI.

Despite the apparent decline in mortality in AKI patients, it remains unacceptably high at around $40 \%$ [1]. If indeed the rising prevalence of $\mathrm{AKI}$ is due to our more 'aggressive' diagnostic and therapeutic approach, then prevention of AKI, both primary and secondary, remains the key to continued improvement in outcome. Ideally, we would like to be able to prevent progression of AKI from milder to more severe forms; therefore, timely intervention is crucial. Aside from how to intervene, the other important question is when to intervene. Over the time course of AKI, just like with sepsis, we can distinguish between a biological and a clinical clock. The first starts when there are alterations in renal perfusion and damage to tubular cells. In contrast, the clinical clock starts only when we see changes in serum creatinine and urine output. Emerging biomarkers of AKI, such as neutrophil gelatinase-associated lipocalcin and cystatin $\mathrm{C}$, give us a view of the biological clock, and the use of commercially available assays for cystatin $\mathrm{C}$ has been increasing [6]. It will, however, take time before this practice becomes universal, particularly in developing countries. Until then, we have to continue to rely on, and improve, the clinical clock. Currently, consensus definitions for AKI exist and are being increasingly used in the literature $[7,8]$. The advent of RIFLE (Risk-InjuryFailure-Loss of renal function-Endstage renal disease) and AKIN (Acute Kidney Injury Network) criteria provides us a framework for identifying and staging AKI. This will not only aid us in recruiting patients, but also serve as clinical endpoints for evaluating interventions in AKI. In the future, outcomes in AKI will include intermediate endpoints, such as prevention in progression from milder to more severe forms of $\mathrm{AKI}$, analogous to what we now do in chronic kidney disease.

\section{Competing interests}

Both authors have participated in the Acute Dialysis Quality Initiative workgroups.

\section{References}

1. Bagshaw SM, George C, Bellomo R, ANZICS Database Management Committee: Changes in the incidence and outcome for early acute injury in a cohort of Australian intensive care units. Crit Care 2007, 11:R68.

2. Ympa YP, Sakr Y, Reinhart K, Vincent JL: Has mortality from acute renal failure decreased? A systematic review of the literature. Am J Med 2005, 118:827-832.
3. Waikar SS, Curhan GC, Wald R, McCarthy EP, Chertow GM: Declining mortality in patients with acute renal failure, 1988 to 2002. J Am Soc Nephrol 2006, 17:1143-1150.

4. Xue JL, Daniels F, Star RA, Kimmel PL, Eggers PW, Molitoris BA, Himmelfarb J, Collins AJ: Incidence and mortality of acute renal failure in Medicare beneficiaries, 1992 to 2001. J Am Soc Nephrol 2006, 17:1135-1142.

5. Lamiere N, Van Biesen W, Vanholder R: The rise of prevalence and the fall of mortality of patients with acute renal failure: what the analysis of two databases does and does not tell us. $J$ Am Soc Nephrol 2006, 17:923-925.

6. Dejavaran P: Emerging biomarkers of acute kidney injury. Contrib Nephrol 2007, 156:203-212.

7. Bellomo R, Ronco C, Kellum J, Mehta R, Palevsky P, the ADQI workgroup: Acute renal failure-definition, outcome measures, animal models, fluid therapy and information technology needs: the Second International Consensus Conference of the Acute Dialysis Quality Initiative (ADOI) Group. Crit Care 2004, 8:R204-R2121.

8. Mehta RL, Kellum JA, Shah SV, Molitoris BA, Ronco C, Warnock DG, Levin A: Acute Kidney Injury Network: report of an initiative to improve outcomes in acute kidney injury. Crit Care 2007, 11:R31. 